\title{
Clinical Efficacy of Endoscopic Submucosal Dissection for Adenocarcinomas of the Esophagogastric Junction
}

Authors

Institutions
Yasuaki Nagami ${ }^{1}$, Hirohisa Machidaa ${ }^{2}$, Masatsugu Shiba ${ }^{1}$, Tomoko Obayashi ${ }^{1}$, Masaki Ominami ${ }^{1}$, Shusei Fukunaga ${ }^{1}$, Satoshi Sugimori ${ }^{1}$, Hirokazu Yamagami ${ }^{1}$, Tetsuya Tanigawa ${ }^{1}$, Kenji Watanabe ${ }^{1}$, Toshio Watanabe ${ }^{1}$, Kazunari Tominaga', Yasuhiro Fujiwara', Tetsuo Arakawa'

${ }^{1}$ Department of Gastroenterology, Osaka City University Graduate School of Medicine, Osaka, Japan

${ }^{2}$ Department of Gastroenterology, Machida Gastrointestinal Hospital, Osaka City, Japan received

3. November 2013 accepted after revision 13. January 2014

\section{Bibliography}

DOI http://dx.doi.org/

10.1055/s-0034-1365276

Published online: 7.3.2014

Endoscopy International Open

2014; 02: E15-E20

(c) Georg Thieme Verlag KG

Stuttgart · New York

E-ISSN 2196-9736

\section{Corresponding author}

Yasuhiro Fujiwara

Department of

Gastroenterology

Osaka City University

Graduate School

1-4-3, Asahimachi,

Abeno-ku, Osaka

Osaka 545-8585

Japan

yasu@med.osaka-cu.ac.jp
Background and Study Aims There are a few reports about the efficacy of endoscopic submucosal dissection (ESD) for adenocarcinomas of the esophagogastric junction (EGJ). However, there is no detailed analysis that divides EGJ cancers into Barrett's adenocarcinoma and gastric cardia adenocarcinoma. The aim of this study was to analyze the efficacy of ESD for EGJ cancers, comparing these two adenocarcinomas.

Patients and Methods This study included 43 patients who underwent ESD for type II EGJ cancers between 2004 and 2011. Pathological examination of resected specimens confirmed 14 cases of Barrett's adenocarcinoma and 29 cases of gastric cardia adenocarcinoma. Cutting margins on the oral side were placed $1 \mathrm{~cm}$ from the squamocolumnar junction, or $1 \mathrm{~cm}$ away from the slight elevation that is an endoscopic sign of subsquamous carcinoma extension. Clinical outcomes, prevalence and length of subsquamous carcinoma ex-

\section{Introduction}

\section{$\nabla$}

Although the incidence of adenocarcinoma of the esophagus and esophagogastric junction (EGJ) is dramatically increasing in Western countries, [1, 2] esophageal adenocarcinoma (Barrett's adenocarcinoma) is uncommon, accounting for only $1 \%-4 \%$ of the esophageal carcinoma in Eastern countries, including Japan. [3,4] Since most cases of Barrett's adenocarcinoma found in Japan arise from short-segment Barrett's esophagus, $[3,5,6]$ it is difficult to distinguish Barrett's adenocarcinoma preoperatively from gastric cardia adenocarcinoma in patients with type II EGJ cancers (tumors invading the EGJ, in which the center is located between $1 \mathrm{~cm}$ above and 2 $\mathrm{cm}$ below the EGJ). [5-7]

Endoscopic submucosal dissection (ESD) is potentially a useful, safe, and curative treatment for superficial gastrointestinal cancers including esophageal, gastric, and colonic cancers. [8-10] There tension, and long-term outcomes were compared between these two types of adenocarcinoma. Results No significant differences in clinical outcomes were found between these two types of adenocarcinoma (en bloc, $100 \%$ versus $100 \%$; complete, $100 \%$ versus $89.7 \%$; curative, $85.7 \%$ versus $75.9 \%$ ). No serious adverse events were encountered. The prevalence of subsquamous carcinoma extension was significantly higher in Barrett's adenocarcinoma compared with gastric cardia adenocarcinoma. Local and distant recurrence were not observed in any cases with curative resection during the follow-up period (1.6-87.6 months).

Conclusion ESD for EGJ cancers, including both Barrett's adenocarcinoma and gastric cardia adenocarcinoma, was efficient and useful. ESD with a $1 \mathrm{~cm}$ safety margin may be acceptable for EGJ cancers.

are a few reports that examine the efficacy of ESD for EGJ cancers, $[5,11-14]$ but there has not been a more detailed analysis that subdivides EGJ cancers into Barrett's adenocarcinoma and gastric cardia adenocarcinoma. Adenocarcinoma of the EGJ often invades underneath the normal esophageal squamous epithelium (subsquamous carcinoma extension). $[3,4]$ This is clinically problematic in EGJ cancers treated with ESD, because cancer margins (especially on the oral side) cannot be accurately determined when subsquamous carcinoma extension is not visible during the preoperative endoscopy.

The aim of the present study was to analyze EGJ cancers retrospectively and compare the efficacy, adverse events, and long-term outcomes of ESD, as well as disease prevalence and length of subsquamous carcinoma extension, between Barrett's adenocarcinoma and gastric cardia adenocarcinoma. The classification of the type of adeno- 


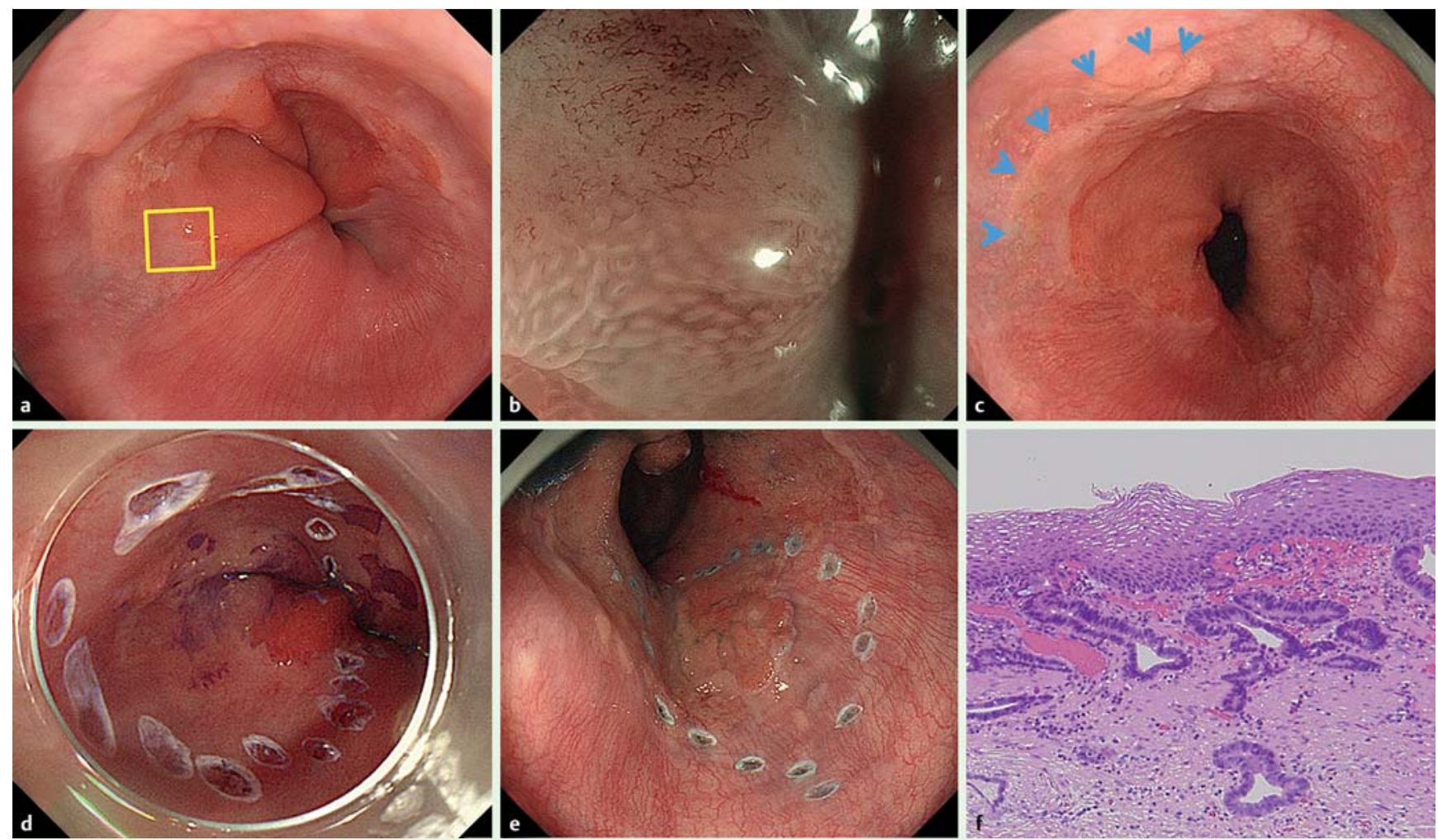

Fig. 1a-f Endoscopic appearance of esophagogastric junction (EGJ) cancers and their histology. a White-light endoscopic image showing a slightly depressed lesion located at the left wall of the EGJ. b Narrow-band imaging magnifying endoscopic image showing the presence of a demarcation line with the presence of an irregular microvascular pattern (*). c Endoscopic appearance of subsquamous carcinoma extension, showing the slight elevation that is similar to the appearance of a submucosal tumor (arrow). $\mathbf{d}$ Marking dots on the oral side of the tumor in a case showing a slight elevation were placed $1 \mathrm{~cm}$ away from the elevation. e Marking dots on the oral side of the tumor in a case not showing a slight elevation were placed $1 \mathrm{~cm}$ away from the squamocolumnar junction. f Histology of a subsquamous carcinoma extension. Hematoxylin-eosin staining. Original magnification $\times 200$.

carcinoma was confirmed by pathological examination of resected specimens.

\section{Patients and Methods \\ $\nabla$}

\section{Patients}

Between 2007 and 2011, 1024 cases of superficial gastric and esophageal cancer were treated with ESD by our department. From this group, 43 (4.2\%) cases of type II EGJ cancer, identified according to Siewert's classification, [15] were retrospectively analyzed. ESD is indicated in cases where preoperative endoscopic evaluation and endoscopic biopsy find EGJ cancer that is a differentiated adenocarcinoma with mucosal invasion. EGJ cancers were classified as either Barrett's adenocarcinoma or gastric cardia adenocarcinoma based on pathological examination after ESD. We compared procedure time, resection rate, adverse event incidence, pathology, and long-term outcomes such as recurrence and survival time between these two groups. The protocol of this study was approved by the Institutional Review Board of the Osaka City University Graduate School of Medicine.

\section{Determination of the Lateral Margin of the Tumor and Setting of Marking before ESD}

Preoperative endoscopy was performed by using an Evis Lucera Spectrum system (Olympus, Tokyo, Japan) with a magnifying upper gastrointestinal endoscope (GIF-H260Z; Olympus). We delineated the area of the lesion using conventional white light endoscopy ( $\bullet$ Fig. 1 a) and magnifying endoscopy with narrow- band imaging (NBI). We assessed the presence of a demarcation line with the presence of an irregular microvascular pattern or irregular microsurface pattern by performing magnifying endoscopy with NBI according to a systematic but simple classification system based on microvascular pattern and microsurface pattern (the VS classification system) [16,17] ( $\bullet$ Fig. $\mathbf{1 b}$ ). An endoscopic sign of subsquamous carcinoma extension is a slight elevation that is similar in appearance to a submucosal tumor located at the esophageal squamous epithelium and continuing from the squamocolumnar junction ( $\bullet$ Fig. 1 c). Marking dots were placed using a bipolar needle knife (B knife; Xemex Co., Tokyo, Japan) or the monopolar needle knife (Flush knife, DK2618JN; Fujifilm Medical, Tokyo, Japan). The marking dots were positioned at least $2 \mathrm{~mm}$ outside the margin of the tumor, except on the oral side, where the marking dots were placed $1 \mathrm{~cm}$ away from the slight elevation ( $\bullet$ Fig. $1 \mathbf{d}$ ), or $1 \mathrm{~cm}$ from the squamocolumnar junction in cases with no abnormal endoscopic findings that were suggestive of subsquamous carcinoma extension ( $\square$ Fig.1 e). $\bullet$ Fig. $1 \mathrm{f}$ demonstrates the histology of subsquamous carcinoma extension.

\section{ESD Procedure}

Experienced endoscopists conducted all endoscopic procedures. Intravenous midazolam and pethidine hydrochloride were used to place patients under conscious sedation for endoscopic procedures. During ESD procedures, a single-channel upper gastrointestinal endoscope with a water jet system (GIF-Q260J; Olympus) with a transparent hood (D-201-11804; Olympus) attached to the tip of the endoscope and a standard electrosurgical generator 
(ICC 200 or VIO300D; Erbe Elektromedizin GmbH, Tübingen, Germany) were used. A B-knife or a Flush Knife and an insulationtipped diathermy knife (IT knife-2; Olympus) were the main electrosurgical knives. A Coagrasper (FD-410LR; Olympus) was also used to stop bleeding or to prevent hemorrhage before vessel cutting. Initially, a hyaluronic acid solution was injected into the submucosal layer around the marking dots to lift it. Next, a needle knife was used to make a circumferential mucosal incision on the oral side around the periphery of the marking dots. After additional submucosal injection below the lesion, submucosal dissection was performed, using the same needle knife, from the oral side toward the anal side. After removing the lesion to the cardia of the stomach, circumferential cutting on the anal side, followed by submucosal dissection, was performed using a retroflex approach with an IT knife-2, and complete en bloc resection was performed. The total procedure time was defined as the period from injection of hyaluronic acid solution to removal of the tumor. Procedure speed $\left(\mathrm{min} / \mathrm{cm}^{2}\right)$ was calculated as the total procedure time $(\mathrm{min})$ divided by the area of the resected specimen $\left(\mathrm{cm}^{2}\right)$.

\section{Pathological Examination}

After fixation in formalin, the resected specimens were cut into 2-mm slices. Histological type, size, depth of invasion, lateral and vertical margins, and lymphovascular invasion were evaluated in each slice. Barrett's adenocarcinoma was defined as cancer arising from Barrett's esophagus, which was defined by the presence of esophageal glands, duplication of the muscularis mucosae under the lesions, or a squamous island within the lesions, in accordance with the Japanese Classification of Esophageal Cancer. [18] Gastric cardia adenocarcinoma was defined as cancer that did not have these characteristics. When adenocarcinoma had subsquamous carcinoma extension, microscopy with a displayed scale was used to determine the length of extension.

\section{Definition of Resection}

An en bloc resection was defined as a resection in one piece that included all markings. A complete resection was defined as an en bloc resection with histologically cancer-free margins. A curative resection of Barrett's adenocarcinoma was defined as a complete resection within an invasion depth of T1a-DMM (deep muscularis mucosa) and without lymphovascular invasions, [18] while curative resection of gastric cardia adenocarcinoma was defined as a complete resection without lymphovascular invasion that fulfilled the following criteria in accordance with the Japanese Classification of Gastric Carcinoma [19]: intramucosal cancer, regardless of tumor size, without ulceration; intramucosal cancer less than $30 \mathrm{~mm}$ in size with ulceration; or minute submucosal cancer (within $500 \mu \mathrm{m}$ from muscularis mucosa) less than $30 \mathrm{~mm}$ in size.

\section{Adverse Events}

Perforation was defined as a visual hole in the esophageal or gastric wall that exposed the mediastinal or peritoneal cavity as recognized on endoscopy. Delayed bleeding was defined as bleeding with hematemesis or melena that required endoscopic reintervention or transfusion after the ESD procedure. Esophageal stricture was defined as diameter reduction with dysphagia that a standard 9.2-mm-diameter endoscope (GIF-Q260; Olympus) failed to pass through.

\section{Follow-up}

For the patients who underwent curative resection, endoscopic examinations were performed 2 and 12 months after ESD, and annual surveillance was performed thereafter. Contrast-enhanced computed tomography was also performed annually to detect lymph node and distant metastasis. For the patients who underwent noncurative resection, additional surgical resection or chemotherapy was recommended, but the choice of additional treatment depended on the case.

\section{Statistical Analysis}

Characteristic values of the enrolled patients and clinical outcomes are presented as the median with the range, the mean \pm standard deviation (SD), or as a percentage. Differences between the groups were examined by using chi-square tests, one-way ANOVA followed by Fisher's paired least-significant difference test, or Student's $t$-test. $P<0.05$ was considered significant. Long-term outcomes were analyzed using the Kaplan-Meier method and log-rank test. Again, $P<0.05$ was considered significant. Statistical analyses were performed using SPSS software version 21.0 for Windows (SPSS Inc., Japan).

\section{Results \\ $\nabla$}

Clinicopathological Characteristics of the Study Subjects Of the 43 cases of EGJ cancer, 14 (32.6\%) were pathologically diagnosed as Barrett's adenocarcinoma while 29 (67.4\%) were gastric cardia adenocarcinoma. One case of Barrett's adenocarcinoma arose from long-segment Barrett's esophagus, while the remaining 13 cases arose from short-segment Barrett's esophagus. The clinicopathological characteristics of the study subjects are shown in Table 1. There were no significant differences in age, gender, tumor size, macroscopic appearance, or depth of cancer invasion between the Barrett's adenocarcinoma group and the gastric cardia adenocarcinoma group. However, the incidence of subsquamous carcinoma extension was significantly higher in Barrett's adenocarcinoma $(12 / 14,85.7 \%)$ than in gastric cardia adenocarcinoma $(2 / 29,6.9 \%)$. The median length of subsquamous carcinoma extension was $3.0 \mathrm{~mm}$, with a range of $0.2 \mathrm{~mm}$ to $7.0 \mathrm{~mm}$, showing that subsquamous carcinoma extension measured less than $1 \mathrm{~cm}$ in all cases. Among the 14 cases of subsquamous carcinoma extension, 6 (42.9\%) were detected by preoperative endoscopy, but $8(57.1 \%)$ were undetected by preoperative endoscopy. There was no significant difference in length

\begin{tabular}{|c|c|c|c|}
\hline & $\begin{array}{l}\text { Barrett's ade- } \\
\text { nocarcinoma }\end{array}$ & $\begin{array}{l}\text { Gastric cardia } \\
\text { adenocarcinoma }\end{array}$ & $P$ value \\
\hline & $n=14$ & $n=29$ & \\
\hline Age, years, mean $\pm S D$ & $61.4 \pm 14.2$ & $65.3 \pm 11.8$ & 0.25 \\
\hline Sex, male/female, n & $13 / 1$ & $24 / 5$ & 0.65 \\
\hline $\begin{array}{l}\text { Tumor size, mm, } \\
\text { median (range) }\end{array}$ & $18(8-30)$ & $13(2-67)$ & 0.65 \\
\hline $\begin{array}{l}\text { Macroscopic } \\
\text { appearance, elevated/ } \\
\text { depressed, } n\end{array}$ & $6 / 8$ & $18 / 11$ & 0.33 \\
\hline $\begin{array}{l}\text { Depth of cancer, } \\
\text { mucosa/submucosa, n }\end{array}$ & $12 / 2$ & $19 / 10$ & 0.28 \\
\hline $\begin{array}{l}\text { Subsquamous carci- } \\
\text { noma extension, n (\%) }\end{array}$ & $12(85.7)$ & $2(6.9)$ & $<0.01$ \\
\hline
\end{tabular}




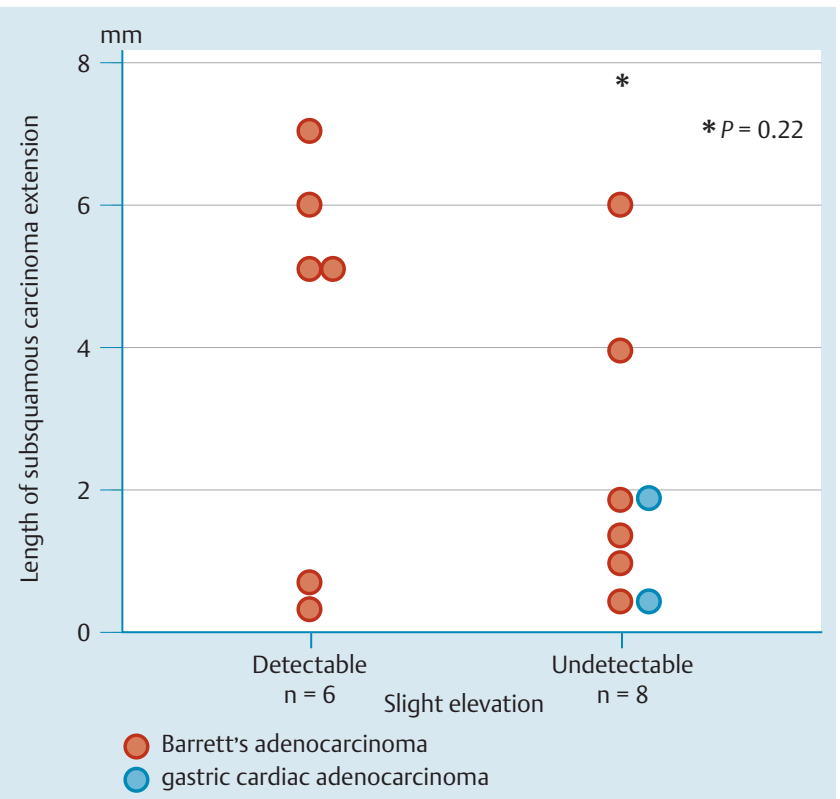

Fig. 2 Length of subsquamous carcinoma extension according to the detection of endoscopic signs. There was no difference in length of carcinoma extension between detectable and undetectable cases of subsquamous carcinoma extension.

between detectable and undetectable cases of subsquamous carcinoma extension ( $\bullet$ Fig. 2 ).

\section{Clinical Outcomes of ESD}

Clinical outcomes and adverse events are shown in $\bullet$ Table 2. Although total procedure times of ESD for Barrett's adenocarcinoma and gastric cardia adenocarcinoma were not significantly different, the procedure speed of ESD for Barrett's adenocarcinoma was significantly faster than the speed for gastric cardia adenocarcinoma $(P=0.03)$. There were no significant differences in en bloc, complete, and curative resection rates between Barrett's adenocarcinoma and gastric cardia adenocarcinoma. All lesions were resected en bloc by ESD. Pathological examination showed that all Barrett's adenocarcinoma and gastric cardia adenocarcinoma lesions were resected with a negative lateral margin, but in two cases of gastric cardia adenocarcinoma that had invaded the submucosa a positive vertical margin was revealed. Twelve cases $(85.7 \%$ ) of Barrett's adenocarcinoma and 22 cases $(75.9 \%)$ of gastric cardia adenocarcinoma were found to have curative resection. Among curatively resected cases of Barrett's adenocarcinoma, five had an invasion depth of T1a-SMM (superficial muscularis mucosa), 2 cases T1a-LPM (lamina propria muosa,), and 5 cases T1a-DMM. [18] Among curatively resected cases of gastric cardia adenocarcinoma, 19 had a mucosal invasion depth and 3 cases a submucosal invasion depth (less than $500 \mu \mathrm{m}, \mathrm{SM} 1$ ) [19]. One case of noncuratively resected Barrett's adenocarcinoma was a tumor with submucosal invasion over $200 \mu \mathrm{m}$ (SM2), while one case was a poorly differentiated adenocarcinoma with submucosal invasion less than $200 \mu \mathrm{m}$ (SM1). [18] On the other hand, all cases of noncuratively resected gastric cardia adenocarcinoma were tumors with submucosal invasion over $500 \mu \mathrm{m}$ (SM2), and 2 cases of these had lymphovascular invasion. [19] There was 1 case $(3.4 \%)$ of procedure-related perforation among the gastric cardia adenocarcinoma group.Postoperative adverse events were delayed bleeding and esophageal stricture, which were each encountered in 1 case (3.4\%) of gastric cardia adenocarcino-
Table 2 Clinical outcome and adverse events of endoscopic submucosal dissection.

\begin{tabular}{|c|c|c|c|}
\hline & $\begin{array}{l}\text { Barrett's } \\
\text { adenocarcinoma }\end{array}$ & $\begin{array}{l}\text { Gastric cardia } \\
\text { adenocarcinoma }\end{array}$ & $P$ value \\
\hline & $n=14$ & $n=29$ & \\
\hline \multicolumn{4}{|l|}{ Procedure } \\
\hline $\begin{array}{l}\text { Total time, min, } \\
\text { median (range) }\end{array}$ & $85(29-176)$ & $124(34-331)$ & 0.11 \\
\hline $\begin{array}{l}\text { Speed, } \min / \mathrm{cm}^{2} \text {, } \\
\text { median (range) }\end{array}$ & $4.9(2.8-16.1)$ & $11.9(1.8-22.3)$ & 0.03 \\
\hline \multicolumn{4}{|l|}{$\begin{array}{l}\text { Results of } \\
\text { resection, n (\%) }\end{array}$} \\
\hline En bloc resection & $14(100)$ & $29(100)$ & 1.00 \\
\hline $\begin{array}{l}\text { Complete } \\
\text { resection }\end{array}$ & $14(100)$ & $27(89.7)$ & 0.54 \\
\hline Curative resection & $12(85.7)$ & $22(75.9)$ & 0.69 \\
\hline \multicolumn{4}{|l|}{$\begin{array}{l}\text { Adverse events, } \mathrm{n} \\
\text { (\%) }\end{array}$} \\
\hline Perforation & $0(0)$ & $1(3.4)$ & 0.48 \\
\hline Delayed bleeding & $0(0)$ & $1(3.4)$ & 0.48 \\
\hline Stricture & $0(0)$ & $1(3.4)$ & 0.48 \\
\hline
\end{tabular}

ma. All patients recovered with endoscopic and conservative treatment such as oral intake restriction and antibiotics. No serious adverse events that required blood transfusion or surgical treatment were encountered.

\section{Additional Treatment and Long-Term Outcomes}

Among the 9 patients who underwent noncurative resection, 2 patients with Barrett's adenocarcinoma and 2 patients with gastric cardia adenocarcinoma underwent salvage surgery, and 2 of these 4 patients died of other causes. Two patients with gastric cardia adenocarcinoma received chemotherapy, and the remaining 3 patients were observed without additional treatment, but no local or distant recurrence was found during the follow-up period (median 41.5 months, range $2-65$ ).

Among the 34 patients who underwent curative resection, there were no local or distant recurrences during a median follow-up period of 42.3 months (range 11.9-77.4) in patients with Barrett's adenocarcinoma and 41.5 months (range $1.6-87.6$ ) in patients with gastric cardia adenocarcinoma. Two patients with gastric cardia adenocarcinoma died of lung cancer $(n=1)$ and esophageal squamous cell carcinoma $(n=1)$. The patient with esophageal cancer was followed for 5 years at our hospital and cancer was discovered at another hospital 79 months after he underwent ESD at our hospital. The 5-year overall survival rate of patients with curative resection was $100 \%$ for Barrett's adenocarcinoma and $88.9 \%$ for gastric cardia adenocarcinoma, which shows no statistical difference (log-rank test 0.74) ( Fig. 3 ). The 5 -year cause-specific survival rate for both Barrett's adenocarcinoma and gastric cardia adenocarcinoma was $100 \%$.

\section{Discussion}

$\nabla$

The indication for endoscopic resection of superficial EGJ cancers, including Barrett's adenocarcinoma, has not been established because the incidence of lymph node metastasis of these cancers is not well known. Although several studies have suggested that ESD and its criteria for curative resection may be indicated for 


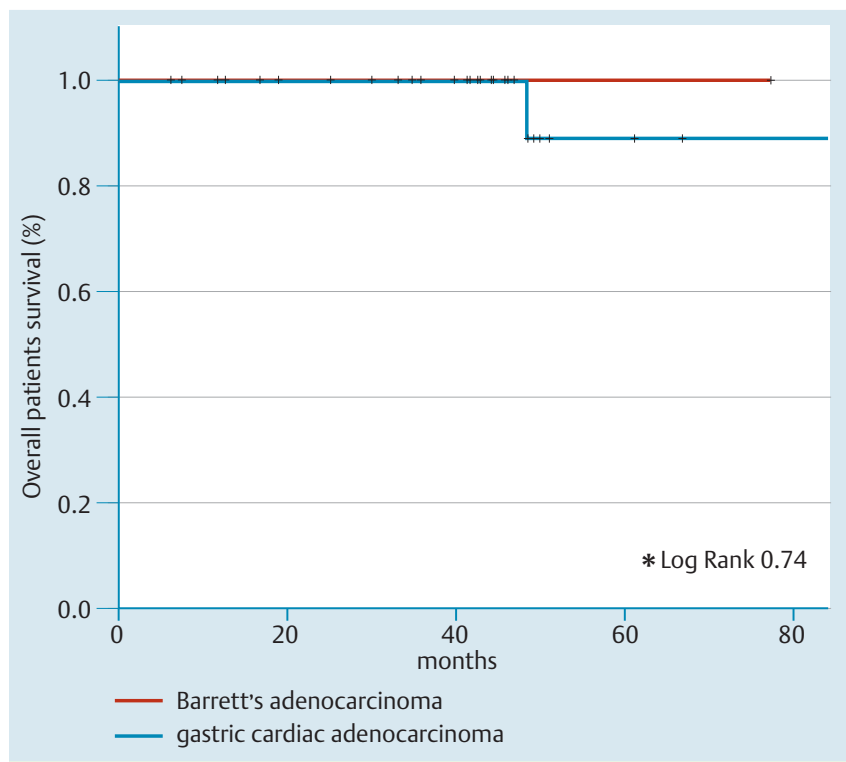

Fig. 3 Overall survival rates in patients with Barrett's adenocarcinoma and those with gastric cardia adenocarcinoma.

EGJ cancers, based on gastric cancer guidelines, [11-14] others have suggested that EGJ cancers have a higher possibility of lymph node metastasis than gastric cancer. [20] Leers et al. reported that the prevalence of lymph node metastasis in Barrett's adenocarcinoma was $1.3 \%$ in patients with mucosal cancer and $22 \%$ in those with submucosal invasion. [21] A systematic review using data from surgical resections showed that 26 of 1350 patients with mucosal Barrett's adenocarcinoma who were treated by surgical resection $(1.93 \%$ ) had lymph node metastasis. [22] These reports suggest that curative resection of Barrett's adenocarcinoma by performing ESD should be defined as resection of cancer limited to the mucosa and not found in the submucosa. This is the reason why we used pathological examination after ESD to divide EGJ cancers into Barrett's adenocarcinoma and gastric cardia adenocarcinoma and evaluated curative resection of these two cancers on the basis of different criteria.

Recently, a large Japanese survey demonstrated that there was no disease-specific death among 111 patients with mucosal Barrett's adenocarcinoma treated by endoscopic resection, and 1 of 32 patients with submucosal invasion $(3.1 \%)$ died because of peritoneal dissemination during an observation period that was longer than 2 years. [3] Since our results showed no recurrence or metastasis in any patient with curative resection, the definition of curative resection used in this study may be acceptable.

Several studies have reported that en bloc resection rates of ESD for EGJ cancers of $90 \%-100 \%$. [11-14, 23] Our results also showed a $100 \%$ en bloc resection rate. The curative resection rates of EGJ cancers according to the Japanese Classification of Gastric Carcinoma are reported to be $72.0 \%-84.1 \%$. [11-14] When Hoteya et al. subdivided EGJ cancers into Barrett's adenocarcinoma and gastric cardia adenocarcinoma, as in our study, they found curative resection rates of $48.0 \%$ for Barrett's adenocarcinoma and $80.6 \%$ for gastric cardia adenocarcinoma. [23] The higher rates of curative resection found in our study might be due to differences in rates of positive lateral margins of the resected specimens. In Hoteya's study, $36 \%$ of Barrett's adenocarcinomas had positive lateral margins due to subsquamous carcinoma extension. [23] However, in our study no cases with positive lateral margins were found because we set $1 \mathrm{~cm}$ safety margins not only in cases in which subsquamous carcinoma extension was detected at preoperative endoscopy, but also in those in whom subsquamous extension was not detected at preoperative endoscopy. In most cases with subsquamous carcinoma extension, the extension was reported to be less than $1 \mathrm{~cm}$; [24] in only 1 case has extension greater than $1 \mathrm{~cm}$ been reported. [25] Therefore, whether an oral safety margin that is placed $1 \mathrm{~cm}$ from the squamocolumnar junction or the slight elevation that is an endoscopic sign of subsquamous carcinoma extension is sufficient or not should be evaluated in the future.

There have been several attempts to detect subsquamous extension of EGJ carcinomas by performing preoperative endoscopy. [4] Goda et al. found that subsquamous carcinoma extension was accurately diagnosed in 97 of 175 Barrett's adenocarcinomas ( $55 \%$ ) by performing conventional white light endoscopy and in 42 of $70(60 \%)$ by performing magnifying endoscopy with NBI, [3] a detection rate similar to that in our study. Recently, Yamagata et al. reported in a small study that magnifying endoscopy with NBI did not provide additional information for the diagnosis of subsquamous carcinoma extension compared with conventional white endoscopy, but acetic acid spraying and magnifying endoscopy with NBI detected $100 \%$ of six patients with subsquamous carcinoma extension. [26] A large study is required to establish the utility of acetic acid spraying and magnifying endoscopy with NBI for the diagnosis of subsquamous carcinoma extension in EGJ cancer.

The results of the present study show that the procedure speed of ESD for Barrett's adenocarcinoma was significantly faster than that of ESD for gastric cardia adenocarcinoma. The exact reasons for this difference are unknown. Tumor location could be a factor, because among type II EGJ cancers gastric cardia adenocarcinoma may extend beyond the cardia, including the angle of His, while Barrett's adenocarcinoma does not. In such cases, it is technically difficult to complete the ESD procedure: the complicated shapes make it difficult to attach the endoknife to mucosa or submucosa. Surgical resection, including total gastrectomy with transhiatal resection and esophagectomy, has been performed for EGJ cancers. Long-term outcomes for superficial cancers were reported to be favorable. Endoscopic therapy was adapted as an alternative therapy for superficial EGJ cancers, because surgical resection has a high mortality rate that often exceeds $2 \%,[27,28]$ with substantial morbidity and no guarantee of curing metastases. Other therapies such as photodynamic therapy, radiofrequency ablation (RFA), endoscopic mucosal resection (EMR), and a combination therapy of EMR and RFA are mainly applied to adenocarcinomas in long-segment Barrett's esophagus, $[2,29,30]$ because Barrett's esophagus has potent malignant potential and the incidence rates of recurrent or metachronous cancers are high, and these therapies enable treatment of both Barrett's adenocarcinoma and Barrett's esophagus. Since most Barrett's adenocarcinoma found in Japan arises from short-segment Barrett's esophagus and metachronous cancer in the Barrett's esophagus is rare, we believe that ESD is an acceptable first-line treatment for superficial EGJ cancers including Barrett's adenocarcinoma.

Our study has some limitations. First, the sample size was small because EGJ cancers are very rare in Japan. The incidence of superficial EGJ cancer in our study was $4.2 \%$, which was comparable to other recent Japanese studies. Second, our study was a retrospective observational analysis, not a randomized clinical trial. A prospective randomized study is required to determine the appropriate positioning of safety margins on the oral side of 
EGJ cancers. Third, this study was a single Japanese expert center experience. The present results may not be applicable to EGJ cancers in Western countries because Barrett's adenocarcinomas in their patients mainly arise from long-segment Barrett's esophagus. In addition the technique of ESD for EGJ cancers requires some expertise.

In conclusion, ESD was efficient and useful for the treatment of superficial EGJ cancers including Barrett's adenocarcinoma and gastric cardia adenocarcinoma. ESD with a $1 \mathrm{~cm}$ safety margin may be effective for EGJ cancers with subsquamous carcinoma extension.

Competing interests: Yes; Tetsuo Arakawa had advisory committees by Otsuka Pharmaceutical Co. Ltd and Eisai Co. Ltd. Yasuhiro Fujiwara had advisory committees by Eisai Co. Ltd. Kenji Watanabe had advisory committees by Mitsubishi Tanabe Pharma Corporation Co. Ltd, Abbott Japan Co. Other authors declare that they have no conflict of interest.

\section{References}

1 Sharma P. Clinical practice. Barrett's esophagus. N Engl J Med 2009; 361: $2548-2556$

2 Bennett C, Vakil N, Bergman J et al. Consensus statements for management of Barrett's dysplasia and early-stage esophageal adenocarcinoma, based on a Delphi process. Gastroenterology 2012; 143: 336-346

3 Goda K, Singh R, Oda I et al. Current status of endoscopic diagnosis and treatment of superficial Barrett's adenocarcinoma in Asia-Pacific region. Dig Endosc 2013; 25: 146 - 150

4 Oyama T. Diagnostic strategies of superficial Barrett's esophageal cancer for endoscopic submucosal dissection. Dig Endosc 2013; 25: 017 12

5 Kakushima N, Yahagi N, Fujishiro $M$ et al. Efficacy and safety of endoscopic submucosal dissection for tumors of the esophagogastric junction. Endoscopy 2006; 38: 170 - 174

6 Kusano C, Gotoda T, Khor CJ et al. Changing trends in the proportion of adenocarcinoma of the esophagogastric junction in a large tertiary referral center in Japan. J Gastroenterol Hepatol 2008; 23: 1662 - 1665

7 Nakamura T, Ide H, Eguchi R et al. Adenocarcinoma of the esophagogastric junction: a summary of responses to a questionnaire on adenocarcinoma of the esophagus and the esophagogastric junction in Japan. Dis Esophagus 2002; 15: 219-225

8 Ono S, Fujishiro M, Niimi $K$ et al. Long-term outcomes of endoscopic submucosal dissection for superficial esophageal squamous cell neoplasms. Gastrointest Endosc 2009; 70: 860-866

9 Isomoto $H$, Shikuwa S, Yamaguchi $N$ et al. Endoscopic submucosal dissection for early gastric cancer: a large-scale feasibility study. Gut 2009; 58: 331 - 336

10 Saito Y, Uraoka T, Yamaguchi Yet al. A prospective, multicenter study of 1111 colorectal endoscopic submucosal dissections (with video). Gastrointest Endosc 2010; 72: 1217-1225

11 Yoshinaga S, Gotoda T, Kusano C et al. Clinical impact of endoscopic submucosal dissection for superficial adenocarcinoma located at the esophagogastric junction. Gastrointest Endosc 2008; 67: 202 - 209

12 Hirasawa K, Kokawa A, Oka $\mathrm{H}$ et al. Superficial adenocarcinoma of the esophagogastric junction: long-term results of endoscopic submucosal dissection. Gastrointest Endosc 2010; 72: 960-966
13 Omae M, Fujisaki J, Horiuchi Y et al. Safety, efficacy, and long-term outcomes for endoscopic submucosal dissection of early esophagogastric junction cancer. Gastric Cancer 2013; 16: 147-154

14 Imai K, Kakushima N, Tanaka $M$ et al. Validation of the application of the Japanese curative criteria for superficial adenocarcinoma at the esophagogastric junction treated by endoscopic submucosal dissection: a long-term analysis. Surg Endosc 2013; 27: 2436 - 2445

15 Siewert J, Stein H. Carcinoma of the cardia: carcinoma of the gastroesophageal junction: classification, pathology and extent of resection. Dis Esophagus 1996; 9: 173-182

16 Yao K. How is the VS (vessel plus surface) classification system applicable to magnifying narrow-band imaging examinations of gastric neoplasias initially diagnosed as low-grade adenomas? Gastric Cancer 2012; $15: 118-120$

17 Nagahama T, Yao K, Maki S et al. Usefulness of magnifying endoscopy with narrow-band imaging for determining the horizontal extent of early gastric cancer when there is an unclear margin by chromoendoscopy (with video). Gastrointest Endosc 2011; 74: 1259-1267

18 The Japanese Esophageal Society. Guidelines for the clinical and pathologic studies on carcinoma of the esophagus. Esophagus 2004; 1: 61 88

19 Japanese Gastric Cancer Association. Japanese gastric cancer treatment guidelines 2010 (ver. 3). Gastric Cancer 2011; 14: 113 - 123

20 van Sandick JW, van Lanschot JJ, ten Kate FJ et al. Pathology of early invasive adenocarcinoma of the esophagus or esophagogastric junction: implications for therapeutic decision making. Cancer 2000; 88: 2429 2437

21 Leers JM, DeMeester SR, Oezcelik A et al. The prevalence of lymph node metastases in patients with T1 esophageal adenocarcinoma a retrospective review of esophagectomy specimens. Ann Surg 2011; 253: $271-278$

22 Dunbar KB, Spechler SJ. The risk of lymph-node metastases in patients with high-grade dysplasia or intramucosal carcinoma in Barrett's esophagus: a systematic review. Am J Gastroenterol 2012; 107: 850 862 (quiz 863)

23 Hoteya S, Matsui A, Iizuka T et al. Comparison of the clinicopathological characteristics and results of endoscopic submucosal dissection for esophagogastric junction and non-junctional cancers. Digestion 2013; 87: 29-33

24 Oyama T, Tomori A, Takahashi A et al. Diagnosis of lateral extension of Barrett's high grade dysplasia using magnifying endoscopy. Stomach Intestine 2011; 46: 1836-1842

25 Tokioka S, Umegaki E, Takeuchi $N$ et al. A case of the Barrett's adenocarcinoma with intramucosal spread extending. Gastroenterological Endoscopy 2010; 52: 402-411

26 Yamagata T, Hirasawa D, Fujita $N$ et al. Efficacy of acetic acid-spraying method in diagnosing extension of Barrett's cancer under the squamous epithelium. Dig Endosc 2012; 24: 309-314

27 Prasad GA, Wu TT, Wigle DA et al. Endoscopic and surgical treatment of mucosal (T1a) esophageal adenocarcinoma in Barrett's esophagus. Gastroenterology 2009; 137: 815-823

28 Pohl H, Sonnenberg A, Strobel $S$ et al. Endoscopic versus surgical therapy for early cancer in Barrett's esophagus: a decision analysis. Gastrointest Endosc 2009; 70: 623-631

29 Neuhaus H, Terheggen G, Rutz EM et al. Endoscopic submucosal dissection plus radiofrequency ablation of neoplastic Barrett's esophagus. Endoscopy 2012; 44: $1105-1113$

30 Konda VJ, Waxman I. Endotherapy for Barrett's esophagus. Am J Gastroenterol 2012; 107: 827-833 